contain something like 100,000 sources per steradian, compared with 600 per steradian for the fourth catalogue and a mere 45 for the third, which was based on measurements made in 1958. One of the most striking of the points made by Sir Martin Ryle last week was that the region of the universe now accessible to the instruments at Cambridge is so great that the outer limits of observation probably correspond to times in the history of an evolving universe at which the formation of galaxies was commonplace.

The instrument being used in the new survey is the one mile telescope. A large tracking array 5,000 feet by 60 feet is used in conjunction with movable instruments in such a way that the combination of the records of successive scans of the same part of the sky can simulate the sensitivity of a much larger telescope. In the parts of the survey so far carried out, the aerial system has been used to track chosen parts of the sky for 12 hours a day in a period of 2 months. The result, at a frequency of $408 \mathrm{MHz}$, is a survey of a patch of sky 4 degrees in diameter. So far, three such patches have been covered with a limit of sensitivity of $0 \cdot 01 \times 10^{-26}$ watts $\mathrm{m}^{-2} \mathrm{sec}^{-1}$. With plausible assumptions about the intensity distribution of the radio frequencies, this corresponds to a limiting flux density twice as great as at the frequency of $178 \mathrm{MHz}$ used in earlier surveys. Each of the three patches of sky so far surveyed appears to include some 200 radio sources.

One of the principal objectives of the work so far has been to determine the variation of the numbers of sources with varying brightness. In practice, this means constructing the relationship between $\log N$ and $\log S$ where $S$ indicates radio flux and $N$ the number of sources with radio flux greater than $S$. It has been necessary to make comparatively rapid surveys of the whole sky so as to pick out comparatively intense radio sources which can be used as a means of calibrating the new survey. Sir Martin Ryle said last week that the initial slope of the curve-corresponding to comparatively intense sources-is essentially the same as the value of -1.8 obtained in earlier surveys, but that the slope progressively decreases in magnitude to about -0.8 . Compared with the predictions of a static Euclidean model of the universe with uniformly distributed radio sources, the new survey shows an excess of sources of intermediate radio flux. Sir Martin Ryle said that the differences between observation and the predictions of an Einstein-de Sitter model and of the steady-state theory are considerable, and great enough to show "that important evolutionary effects are taking place". He explained that "The observations show a distribution in which there is an apparent increase in the space density of sources followed by a marked decrease. The gradient of the decrease may be determined by the spread in intrinsic luminosities and, if this is as great as that found for the intense sources, the cut-off must be very rapid." This cut-off probably corresponds to the "epoch of galaxy formation".

\section{Binocular Vision in Cats}

\section{from our Neurophysiology Correspondent}

JuLEsz has recently shown that depth perception in human vision does not depend on the existence of monocular cues but that it can be based simply on disparities between the two retinal images (see Nature, 215, 1225; 1967). Barlow, Blakemore and Pettigrew (J. Physiol., 193, 327; 1967) have now set out to discover the mechanisms involved in depth perception in anaesthetized cats. The nub of their conclusion is that in cats, as in men, the recognition of structure usually precedes the investigation of depth but that this sequence of events is not essential.

The technique is to record from single cells in the primary visual cortex, stimulating them either with moving black and white bars or with the edges between black and white areas. Having found the gross receptive field for a single cell, the receptive field is determined for each retina separately. The next step is to determine the disparity between the responses to two identical stimuli, presented binocularly, which produce the greatest excitation of the cortical unit.

For each cat they found a range of disparity of about $6^{\circ}$ in the horizontal plane and $2^{\circ}$ in the vertical. This is interesting, because it is similar to Julesz's result. He found that man could maintain fusion of a stereoscopic pair of images as they were pulled apart on the retinae for a distance in the horizontal three times greater than that in the vertical direction. Precise measurements of retinal disparity are extremely difficult, even in an anaesthetized preparation, because of residual eye movements, and the impossibility of determining the direction of each optic axis accurately. Barlow et al., however, reduced possible movements by paralysing the preparation with 'Flaxedil' and attaching the two eyes to firmly fixed rings. Ophthalmoscopic observation during the experiments showed no perceptible changes in eye position. In any case, the most important feature of their results is the range of disparities observed for each cat, coupled with the horizontal-vertical anisotropy. They suggest that their results indicate that the cat observes depth over a wide range, but at low accuracy (because of poor depth of focus in the visual field), unlike man, who perceives depth accurately over a short range. This seems rather unlikely, both on behavioural grounds and because there would seem to be little advantage in seeing depth in out-of-focus objects. Their results could equally well be taken to show that cats, like humans, can accommodate a large range of depths, or retinal disparities at the same distance and that each part of this range is served by a particular population of cortical neurones. It would be interesting to know whether cats could recognize structures in Julesz patterns: in other words, it would be interesting to know whether cats need to recognize structure monocularly before they can determine the retinal disparities leading to depth percepts. At the moment there is no evidence against this, although Barlow and his colleagues say that their results suggest that the cat recognizes primitive structure first and then decides on the appropriate pairing of parts from the fields of vision of the two eyes.

One of the most important by-products of these experiments is the renewed emphasis on the need for very precise control of eye movements, refractive correction and stimulus presentation in studies on the visua] system. Indeed, this is one of the few reports in this field where adequate care has obviously been taken. 